Helgoländer wiss. Meeresunters. 22, 442-453 (1971)

\title{
Antimikrobielle Inhaltsstoffe in Algen
}

\author{
4. Mitteilung \\ Wirkung der Acrylsäure auf Atmung und Makromolekülsynthese \\ bei Staphylococcus aureus und Escbericbia coli \\ K.-W. Glombitza und R. HeyseR* \\ Pharmakognostisches Institut der Universität Bonn; \\ Bonn, Bundesrepublik Deutscbland
}

\begin{abstract}
Antimicrobial components in algae. 4th contribution. Effect of acrylic acid on respiration and macromolecular synthesis in Stapbyloccus aureus and Escbericbia coli. Among the simple unsaturated carbonic acids, acrylic acid is a comparatively specific inhibitor for Staphylococcus aureus, Escberichia coli and other bacteria. Insignificant bactericidal action can only be observed at high concentrations and long incubation times of acrylic acid. In older $E$. coli cells, acrylic acid inhibits respiration more strongly than growth. On the other hand, in younger $E$. coli cells and in St. aureus, inhibition of growth is more pronounced than that of respiration. Antibacterial activities of acrylic acid against $E$. coli can be reduced by compounds with thiol groups; they can be increased by 2.4-dinitrophenol (DNP). The effect of acrylic acid and DNP is synergistic; such effects are very small in St. aureus. Incorporation of leucine into the protein of $E$. coli cells is less inhibited by acrylic acid than their growth rate; incorporation of thymidine into DNA is inhibited to the same extent as growth; incorporation of uracil into RNA is inhibited to a much higher extent right from the beginning of the experiment. DNP is capable of increasing the degree of inhibition of uracil incorporation caused by acrylic acid. Syntheses of r-RNA and s-RNA are inhibited to nearly the same extent; there seems to be no influence on the synthesis of m-RNA.
\end{abstract}

\section{EINLEITUNG}

Viele marine Grünalgen sowie einige Rotalgen enthalten $z$. T. beträchtliche Mengen freier oder freisetzbarer Acrylsäure (GLombITza 1968). Acrylsäure ist, wie schon Sieburth (1960) für die Chrysophycee Phaeocystis pouchetii beweisen konnte, häufig die Ursache der antimikrobiellen Eigenschaften der Algen.

Die Acrylsäure (Acs) ist nach Befunden von BRIsou \& RAu'tLIN DE LA RoY (1964) bei Escherichia coli und Stapbylococcus aureus wieder auswaschbar; die Hemmwirkung ist somit reversibel. Die Hemmintensität ist stark pH-abhängig (LücK 1959, SIEBURTH 1961). Sie nimmt mit steigender $\mathrm{H}^{+}$-Konzentration bis zum pK-Wert der Säure zu (HACKETT 1960).

\footnotetext{
* Teil der Dissertation von R. Heyser, Bonn, 1970 (D5).
} 
Vereinzelte Angaben zeigen, daß Acs an verschiedenen Stellen des Bakterienstoffwechsels wirksam werden kann. THrusse (1964) fand, daß Acs besonders die Oxydation der Fettsäuren bei Pseudomonas hemmt. WhItLey \& Ordal (1954) beobachteten, daß die Propionatbildung aus Pyruvat bei Micrococcus lactilyticus in Gegenwart von Acs verlangsamt ist. Lück (1959) berichtet über eine Hemmung der Katalase durch Acs, und MrCHEL (1965) untersuchte den Einfluß der Acs auf die intestinalen Bakterien junger Schweine. Er fand eine Verstärkung der Milchsäurebildung und eine Hemmung des Abbaues von Harnstoff, L-Arginin und L-Lysin.

Die Aufgabe der vorliegenden Untersuchung war es festzustellen, inwieweit Störungen der Atmung und bei der Makromolekülsynthese für die bei $E$, coli and St. aureus beobachteten antimikrobiellen Wirkungen der Acs verantwortlich sein können.

\section{MATERIAL UND METHODEN}

B a kterie n : Staphylococcus aureus SG 511, Echerichia coli $\mathrm{G} 1$ (Wildstamm), Bacillus subtilis ATCC 6633, Bacillus cereus A'TCC 9643, Sarcina lutea ATCC 9341, Proteus vulgaris (Wildstamm) und Escherichia coli G-1-Pyruvatstamm.

Anzucht und Keimzahlbestimmung: $100 \mathrm{ml} \mathrm{Nährlösung} \mathrm{(Difco}$ Antibiotic Medium III oder Standard-I-Nährboullon Merck) wurden mit einer Ose voll einer Schrägagarkultur beimpt und bei $37^{\circ} \mathrm{C}$ in Schüttelthermostaten bebrütet. Im Abstand von 30 bzw. 60 min wird in einer entnommenen Probe im Bausch \& LombSpectronic 20 die optische Dichte bei $600 \mathrm{~nm}$ gemessen und gegebenenfalls die Zahl der vermehrungsfähigen Keime/ml auf Difco-Antibiotic-Medium I $(\mathrm{pH} 7,0)$ nach dem KocHschen Plattenverfahren ermittelt (JANKe \& Dickscheit 1967). Aus dieser Anzuchtkultur wird soviel entnommen, wie notwendig ist, um in den Testansätzen eine Einsaat von $10^{6} \mathrm{Keimen} / \mathrm{ml}$ zu erhalten.

Herstellen der Acrylsäurelösung: Die mit Hydrochinon stabilisierte Acs wird unmittelbar vor Gebrauch im Vakuum $\left(\mathrm{Kp}_{11}, 39-40^{\circ} \mathrm{C}\right)$ destilliert und mit $\mathrm{NaHCO}_{3}$ und $0,1 \mathrm{n} \mathrm{NaOH}$ auf den gewünschten $\mathrm{pH}$-Wert eingestellt.

Versuche zur Aufhebung der antibakteriellen Aktivi$\mathrm{t} \ddot{\mathrm{t}} \mathrm{der}$ Acrylsäure: Jeweils 5 Erlenmeyerkolben zu $300 \mathrm{ml}$ werden mit je $90 \mathrm{ml}$ Difco-Antibiotic-Medium III ( $\mathrm{pH} \mathrm{6,5)} \mathrm{beschickt,} \mathrm{beimptt} \mathrm{und} \mathrm{bei} 37^{\circ}$ in Schüttelthermostaten bebrütet. Sobald ein $E_{600}$ von ca. $0,1-0,15$ erreicht ist, gibt man in 4 Kolben Acs bzw. Acs zuzüglich den Testsubstanzen (Endkonzentration an Acs: $412 \gamma / \mathrm{ml}$ bei $E$. coli, $33 \gamma / \mathrm{ml}$ bei St. aureus) in $25 \mathrm{ml}$ Pufferlösung. Man mißt in Abständen von $20 \mathrm{~min}$ die Extinktion bei $600 \mathrm{~nm}$.

Bestimmung der minimalen Hemmkonzentration (MHK): Die MHK wird mit Lösungen frisch destillierter und neutralisierter Acs, Methacrylsäure, Vinylessigsäure, Crotonsäure im Reihenverdünnungstest in der von KLEIN (1957) angegebenen Weise im Difco-Antibiotic-Medium III bei einer Inkubationszeit von 24 Stunden bestimmt.

Untersuchung der Atmung im Warburg-Apparat: Die Atmung wurde in der mehrfach beschriebenen Weise im Warburg-Apparat bei $37^{\circ} \mathrm{C}$, einer Schüttelgeschwindigkeit von 100 Ausschlägen/min und Ableseintervallen von 5 oder 
10 min bestimmt. Die zweiarmigen Gefäße mit Zentralzylinder enthielten z. T. im Zentralzylinder $\mathrm{KOH}(10 \%, 0,2 \mathrm{ml}$ auf Filterpapier), in einem Seitenarm die benötigte Menge Acs in konzentrierter Lösung $(0,2 \mathrm{ml})$ im anderen $\mathrm{HCl}$ bzw. $\mathrm{H}_{2} \mathrm{O}, 2 \mathrm{ml}$ Nährlösung im Hauptraum enthielten zu Versuchsbeginn $10^{6} \mathrm{Keime} / \mathrm{ml}$. Es wurden $\mathrm{O}_{2}-$ Verbrauch und $\mathrm{CO}_{2}$-Entwicklung gemessen.

Einfluß von Acrylsäure auf den Einbau von ${ }^{14} \mathrm{C}-\mathrm{Uracil}$ und ${ }^{14} \mathrm{C}-\mathrm{Thymidin} \mathrm{bzw}$. ${ }^{14} \mathrm{C}-\mathrm{Leucin}:$ E. coli-Pyruvatstamm wird in Nährlösung pH 5,6 (REHм 1967, modifiziert) bis zu einer Dichte von $\mathrm{E}_{600} 0,1$ bzw. 0,5 kultiviert. Je $20 \mathrm{ml}$ dieser Kultur werden mit $1 \mathrm{ml}$ Acs-Lösung (Endkonzentrationen siehe Tabellen 5 und 6) versetzt und in Schüttelthermostaten bei $37^{\circ} \mathrm{C}$ inkubiert. Man mißt nach 5, 15, 25 und 35 Minuten die optische Dichte bei $600 \mathrm{~nm}$. Nach 0, 10, 20 und 30 Minuten entnimmt man 3 Proben von je $1 \mathrm{ml}$ und gibt sie in vorgewärmte $\mathrm{Kölb}$ chen, die entweder $0,5 \mu \mathrm{C}^{14} \mathrm{C}_{(2)}$-Uracil oder $0,5 \mu \mathrm{C}^{14} \mathrm{C}_{(2)}$-Thymidin oder $0,5 \mu \mathrm{C}^{14} \mathrm{C}_{(\mathrm{u})}$ Leucin oder $0,1 \mu \mathrm{C}{ }^{14} \mathrm{C}_{(\mathrm{u})}$-Leucin $+6,8 \gamma$ unmarkiertes Leucin (für $\mathrm{E}_{600}=0,5$ ) enthalten. Nach $5 \mathrm{~min}$ bei $37^{\circ} \mathrm{C}$ gibt man $1 \mathrm{ml}$ 6prozentige Perchlorsäure zu, kühlt $30 \mathrm{~min}$ im Eisbad und nutscht die mit Uracil bzw. Thymidin inkubierten Proben auf Membranfiltern (Sartorius Membranfilter Kat.-Nr. 1311, Poren 0,01 $\mu$ ) ab und wäscht mit $12 \mathrm{ml} 3$ prozentiger kalter $\mathrm{HClO}_{4}$. Die mit Leucin inkubierten Proben werden vor dem Abnutschen $20 \mathrm{~min}$ auf $90^{\circ} \mathrm{C}$ erhitzt. Die Filter werden auf Aluminiumschälchen aufgeklebt und im Methandurchflußzähler die Impulse ausgezählt.

Isolierung der RNS aus E. coli-Pyruvatstamm: E. coliPyruvatstam wird in Pyruvatnährlösung bis zu $\mathrm{E}_{600} 0,5$ kultiviert. $\mathrm{Zu} 200 \mathrm{ml}$ dieser Suspension gibt man $10 \mu \mathrm{C}{ }^{14} \mathrm{C}_{(2)}$-Uracil $+800 \gamma$ unmarkiertes Uracil (Kontrolle) bzw. $30 \mu \mathrm{C}^{14} \mathrm{C}_{(2)}$-Uracil $+800 \gamma$ unmarkiertes Uracil $+50 \mathrm{mg}$ Acs (gehemmte Probe). Man inkubiert $60 \mathrm{~min}$ in Schüttelthermostaten bei $37^{\circ} \mathrm{C}$, fügt $40 \mathrm{mg}$ unmarkiertes Uracil zu und inkubiert wiederum $10 \mathrm{~min}$ bei $37^{\circ} \mathrm{C}$. Man kühlt rasch herunter, zentrifugiert bei $2750 \times \mathrm{g} \mathrm{kalt}$ ab und wäscht zweimal mit 0,5 prozentiger $\mathrm{KCl} / 0,5-0 / 0^{-}$ $\mathrm{NaCl}$-Lösung. Die RNS wird nach der leicht modifizierten Methode von SMON \& PraAg (1964) unter Anwendung von DNase und Extraktion mit Phenol isoliert. Die zuletzt mit Alkohol ausgefällte RNS wird in TM-Puffer $(0,01-\mathrm{m}$-Tris-Puffer pH 8,0 mit $1 \cdot 10^{-4} \mathrm{~m} \mathrm{MgCl}_{2}$ ) aufgenommen und am Saccharosegradienten aufgetrennt. Stufengradient mit Stufen von $1,5 \%$ beginnend mit $20 \%$, endend mit $3 \%$; Zentrifugation 7 Stunden bei $+3^{\circ}$ und $160000 \times \mathrm{g}$ in der Spinco SW 50. Die PolyäthylenZentrifugenröhrchen werden mit einer Kanïle angestochen und 52 bis 54 Fraktionen von je 5 Tropfen gesammelt. Jede zweite Fraktion wird mit $1 \mathrm{ml}$ TM-Puffer pH 8,0 verdünnt und die optische Dichte bei $\mathrm{E}_{260}$ bestimmt. Die übrigen Fraktionen werden mit $2 \mathrm{ml} \mathrm{0,25} \mathrm{m}$ Perchlorsäure behandelt, über Membranfilter abgesaugt und die Im$\mathrm{pulse} / \mathrm{min}$ im Methandurchllußzähler bestimmt.

\section{ERGEBNISSE}

Die minimale Hemmkonzentration (MHK) der Acrylsäure bei pH 6,5 liegt relativ hoch (Tab. 1). Andere mit Acs nahe verwandte bzw. homologe ungesättigte Säuren mit vergleichbaren $\mathrm{pK}$-Werten, wie Methacrylsäure, Vinylessigsäure und Crotonsäure, sind jedoch noch um Zehnerpotenzen weniger wirksam. 
Tabelle 1

Minimale Hemmlonzentration für Acrylsäure und honologe Säuren (in $\mathrm{mg} / \mathrm{ml}$ ). Messung der MHK im Reihenverdünnungstest bei $\mathrm{pH}$ 6,5 in Standkulturen

\begin{tabular}{|lcccccc|}
\hline Säure & $\begin{array}{c}\text { Bacillus } \\
\text { subtilis }\end{array}$ & $\begin{array}{c}\text { Bacillus } \\
\text { cereus }\end{array}$ & $\begin{array}{c}\text { Stapby- } \\
\text { lococcus } \\
\text { aureus }\end{array}$ & $\begin{array}{c}\text { Sarcina } \\
\text { lutea }\end{array}$ & $\begin{array}{c}\text { Eschericbia } \\
\text { coli }\end{array}$ & $\begin{array}{c}\text { Proteus } \\
\text { vulgaris }\end{array}$ \\
\hline Acrylsäure & 0,195 & 0,780 & 0,050 & 25 & 0,78 & 3,1 \\
Methacrylsäure & 25 & 12,5 & 6,25 & 12,5 & 50 & 50 \\
Vinylessigsäure & 12,5 & 12,5 & 6,25 & 12,5 & 25 & 12,5 \\
Crotonsäure & 12,5 & 25 & 12,5 & 12,5 & 25 & 25 \\
\hline
\end{tabular}

Die Hemmwirkung scheint demnach auf einer für Acrylsäure relativ spezifischen Eigenschaft zu beruhen.

Nach BRIsou \& RAUTLIN DE LA RoY (1964) hat Acs nur bakteriostatische Eigenschaften. Bestimmt man die Zahl der kultivierbaren Keime, so ist nach zweistündiger Einwirkungszeit der mehrfachen MHK keine und nach 24stündiger höchstens eine geringfügige Verminderung der Keimzahl feststellbar (Tab. 2).

Tabelle 2

Keimzablbestimmung bei Escherichia coli und Stapbylococcus aureus nach unterschiedlicher Einwirkungszeit von Acrylsäure in Schüttelkulturen (Keime $\times 10^{-7} / \mathrm{ml}$ )

\begin{tabular}{|c|c|c|c|c|c|c|}
\hline \multirow[t]{2}{*}{$\gamma$ Acrylsäure/ml } & \multicolumn{3}{|c|}{$\begin{array}{c}\text { E. coli } \\
\text { Einwirkungszeit }\end{array}$} & \multicolumn{3}{|c|}{$\begin{array}{c}\text { St. aureus } \\
\text { Einwirkungszeit }\end{array}$} \\
\hline & 0 Std & $2 \mathrm{Std}$ & $24 \mathrm{Std}$ & O Std & $2 \mathrm{Std}$ & $24 \mathrm{Std}$ \\
\hline 0 & 7,3 & 87 & 170 & 2,3 & 6,4 & 16 \\
\hline 10 & 7,3 & 73 & 160 & 2,3 & 5,9 & 22 \\
\hline 100 & 7,3 & 51 & 86 & 2,3 & 3,2 & 8,7 \\
\hline 1000 & 7,3 & 9,9 & 33 & - & - & - \\
\hline 10000 & 7,3 & 7,8 & 2,2 & - & - & - \\
\hline
\end{tabular}

Die höhere Empfindlichkeit von St. aureus gegen Acs wie auch die starke pH-Abhängigkeit der zur Hemmung benötigten Dosis ließen sich nicht nur bei der Bestimmung der $\mathrm{MHK}$, sondern auch der Hemmkonzentrationen bestätigen, die zur 50prozentigen bzw. 75 prozentigen Hemmung $\left(\mathrm{HK}_{50}, \mathrm{HK}_{75}\right)$ führten (Tab. 3).

Tabelle 3

$\mathrm{HK}_{50}$ und $\mathrm{HK}_{75}$ bei Staphylococcus aureus und Eschericbia coli in Abhängigkeit vom $\mathrm{pH}$-Wert (in $\gamma$ Acrylsäure/ml)

\begin{tabular}{|cccc|}
\hline $\begin{array}{c}\text { Hemm- } \\
\text { konzentration }\end{array}$ & $\mathrm{pH}$ & E. coli & St. aureus \\
\hline $\mathrm{HK}_{50}$ & 5,6 & 90 & 10 \\
$\mathrm{HK}_{75}$ & 7,5 & 820 & 70 \\
& 5,6 & 125 & 13 \\
\hline
\end{tabular}


Die Werte der Tabelle 3 gelten jedoch nur, wenn die Acs zu Beginn der log.Phase zugesetzt wurde. Während der log-Phase sind höhere Acs-Mengen notwendig, um gleichartige Hemmungen zu erzielen.

\section{Antagonisten der Wirkung der Acrylsäure}

Die Wirkung der Acrylsäure wird bei $E$. coli in geringem Maße durch Nikotinsäureamid, $\mathrm{Mg}^{++}$und sehr deutlich durch $\mathrm{SH}$-haltige Verbindungen, wie Cystein, Penicillamin und Cysteamin, aufgehoben. Bei St. aureus liegen die Verhältnisse etwas anders. Hier haben $\mathrm{Ca}^{++}$und $\mathrm{Mg}^{++}$und $\mathrm{Cysteamin}$ eine schwach antagonistische Wirkung; Cystein und Penicillamin sind unwirksam, Pantothensäure verstärkt die Hemmung.

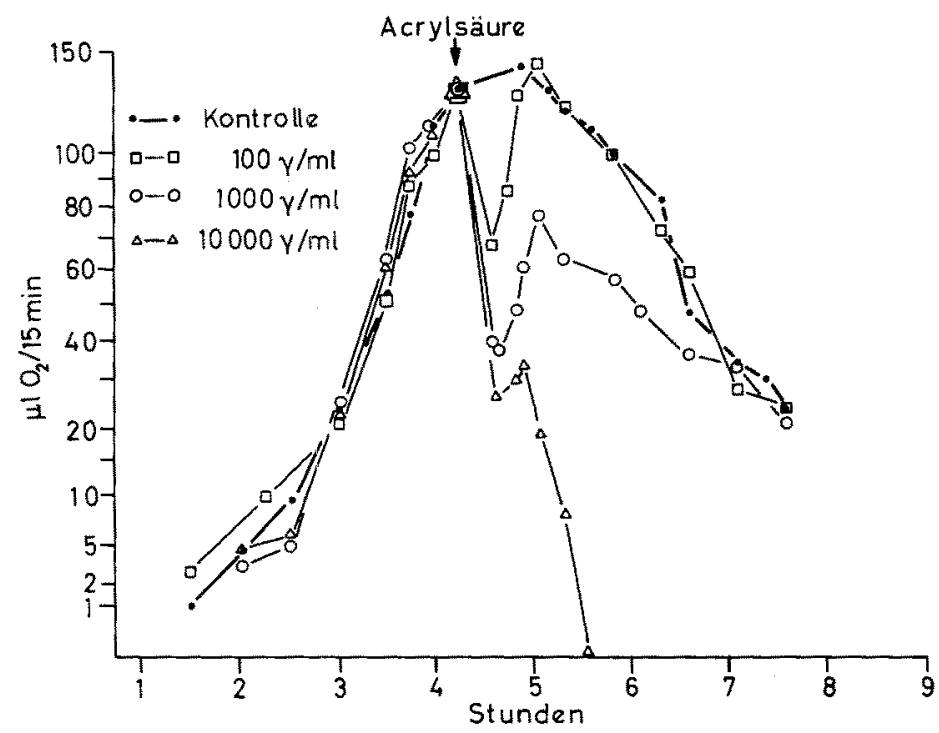

Abb. 1: $\mathrm{O}_{2}$-Verbrauch von Escherichia coli-Zellen bei Zugabe von Acrylsäure (Pfeil) gegen Ende der logarithmischen Phase

2,4-Dinitrophenol wirkt bei $E$. coli sehr stark synergistisch, bei St. aureus ist der Effekt nicht so ausgeprägt. $5 \cdot 10^{-5} \mathrm{~m}$ DNP - ohne Acs - haben bei E. coli gar keine Hemmwirkung, bei St. aureus stören sie das Wachstum in gleichem Umfang wie $33 \gamma$ Acs $/ \mathrm{ml}\left(4,6 \cdot 10^{-4} \mathrm{~m}\right) \cdot 5 \cdot 10^{-4} \mathrm{~m}$ DNP hemmen bei beiden Organismen etwa ebenso stark wie die in den Versuchen zum Einsatz gekommene Acs-Menge (Tab. 4).

Die eingesetzte Acs-Menge entsprach etwa einer $\mathrm{HK}_{50}$ bei $\mathrm{pH}$ 6,5. Verfolgt man die Zunahme der optischen Dichte nach Acs-Zugabe, so beobachtet man bei E. coli eine rasche, fast ohne Latenzphase einsetzende Hemmung, von der sich die Kulturen je nach der Menge der zugegebenen Acs wieder erholen können. Die Ursache liegt nicht in einer Zerstörung des Hemmstoffes (GLOMBITZA 1970, unveröffentlichte Ergebnisse). 


\section{Tabelle 4}

Einfluß verschiedener biologisch aktiver Substanzen auf die Wachstumshemmung bei Escherichia coli und Staphylococcus aureus durch Acrylsäure. Die Prozentwerte beziehen sich auf die beschleunigte Zunahme (positive Werte) oder verlangsamte Zunahme (negative Werte) der optischen Dichte einer Schüttelkultur im Vergleich zur ebenfalls mit Acs gehemmten Kontrolle. Acrylsäure $\left(412 \gamma / \mathrm{ml}=5,7 \cdot 10^{-3} \mathrm{~m}\right.$ bei $E$. coli und $33 \gamma / \mathrm{ml}=4,6 \cdot 10^{-4} \mathrm{~m}$ bei St. atrews) und zu pruffender Stoff wurden gleichzeitig zu Beginn der log.-Phase in einer solchen Konzentration zugegeben, daß in der Nährlösung die in Molaritäten angegebenen Konzentrationen erreicht wurden $(\mathrm{pH} 6,5)$

\begin{tabular}{|c|c|c|c|c|c|}
\hline \multirow{2}{*}{\multicolumn{2}{|c|}{$\begin{array}{l}\text { Steigerung bzw. Verminderung des } \\
\text { Wachstums in } \% \text { zur gehemmten } \\
\text { Kontrolle bei } \\
\text { Minuten nach Zugabe von Acrylsäure } \\
\text { und Testsubstanz }\end{array}$}} & \multicolumn{2}{|c|}{ E. coli } & \multicolumn{2}{|c|}{ St. aureus } \\
\hline & & $0-60$ & $60-120$ & $0-60$ & $60-120$ \\
\hline \multirow{2}{*}{\multicolumn{2}{|c|}{$\begin{array}{ll}\text { Alanin } & 5 \cdot 10^{-5}, 5 \cdot 10^{-4}, 5 \cdot 10^{-3} \mathrm{~m} \\
\text { Biotin } & 5 \cdot 10^{-5}, 5 \cdot 10^{-4}, 5 \cdot 10^{-3} \mathrm{~m}\end{array}$}} & 0 & 0 & 0 & 0 \\
\hline & & 0 & 0 & 0 & 0 \\
\hline \multirow{2}{*}{$\begin{array}{l}\text { Ca-Pantothenat } \\
(+20 \text { mg Na-citrat })\end{array}$} & $5 \cdot 10^{-5} \mathrm{~m}$ & 0 & 0 & -2 & 0 \\
\hline & $5 \cdot 10^{-4} \mathrm{~m}$ & 0 & 0 & -6 & -13 \\
\hline & $5 \cdot 10^{-3} \mathrm{~m}$ & 0 & 0 & -12 & -26 \\
\hline \multirow[t]{5}{*}{ Nikotinsäureamid } & $8,2 \cdot 10^{-5} \mathrm{~m}$ & - & - & 0 & 0 \\
\hline & $4,1 \cdot 10^{-5} \mathrm{~m}$ & - & - & 0 & 0 \\
\hline & $8,2 \cdot 10^{-4} \mathrm{~m}$ & 2 & 8 & 0 & 4 \\
\hline & $4,1 \cdot 10^{-3} \mathrm{~m}$ & 6 & 11 & - & - \\
\hline & $8,2 \cdot 10^{-3} \mathrm{~m}$ & 12 & 16 & - & - \\
\hline \multirow[t]{2}{*}{$\mathrm{CaCl}_{2}$} & $5 \cdot 10^{-5} \mathrm{~m}$ & 0 & 0 & 18 & 10 \\
\hline & $5 \cdot 10^{-4} \mathrm{~m}$ & 11 & -10 & 29 & 29 \\
\hline \multirow[t]{3}{*}{$\mathrm{MgSO}_{4}$} & $5 \cdot 10^{-5} \mathrm{~m}$ & 0 & -5 & 10 & 3 \\
\hline & $5 \cdot 10^{-4} \mathrm{~m}$ & 0 & 25 & 10 & 3 \\
\hline & $5 \cdot 10^{-3} \mathrm{~m}$ & 5 & 33 & -8 & -13 \\
\hline \multirow[t]{5}{*}{ Cystein } & $8,2 \cdot 10^{-5} \mathrm{~m}$ & - & - & 7 & -2 \\
\hline & $4,1 \cdot 10^{-4} \mathrm{~m}$ & - & - & -5 & \\
\hline & $8,2 \cdot 10^{-4} \mathrm{~m}$ & 23 & 65 & -5 & -3 \\
\hline & $4,1 \cdot 10^{-3} \mathrm{~m}$ & 20 & 67 & - & - \\
\hline & $8,2 \cdot 10^{-3} \mathrm{~m}$ & - & 31 & - & - \\
\hline \multirow[t]{5}{*}{ Penicillamin } & $6,7 \cdot 10^{-5} \mathrm{~m}$ & - & - & -10 & 5 \\
\hline & $3,3 \cdot 10^{-4} \mathrm{~m}$ & - & - & -4 & 7 \\
\hline & $6,7 \cdot 10^{-4} \mathrm{~m}$ & 21 & 100 & - & 一 \\
\hline & $3,3 \cdot 10^{-3} \mathrm{~m}$ & 27 & 93 & - & - \\
\hline & $6,7 \cdot 10^{-3} \mathrm{~m}$ & 19 & 158 & - & - \\
\hline \multirow[t]{6}{*}{ Cysteamin } & $1,3 \cdot 10^{-4} \mathrm{~m}$ & - & - & 0 & 2 \\
\hline & $6,5 \cdot 10^{-4} \mathrm{~m}$ & - & - & 5 & 5 \\
\hline & $3,2 \cdot 10^{-4} \mathrm{~m}$ & 5 & 62 & - & - \\
\hline & $6,5 \cdot 10^{-4} \mathrm{~m}$ & - & - & 11 & 14 \\
\hline & $1,3 \cdot 10^{-3} \mathrm{~m}$ & 20 & 131 & - & - \\
\hline & $6,5 \cdot 10^{-3} \mathrm{~m}$ & 41 & 154 & - & - \\
\hline \multirow[t]{3}{*}{ DNP } & $5 \cdot 10^{-6} \mathrm{~m}$ & -11 & -5 & - & - \\
\hline & $5 \cdot 10^{-5} \mathrm{~m}$ & -11 & -9 & -6 & 8 \\
\hline & $5 \cdot 10^{-4} \mathrm{~m}$ & -77 & -90 & -39 & -29 \\
\hline
\end{tabular}

\section{Einfluß der Acrylsäure auf die Atmung}

Mißt man im Warburg-Apparat den $\mathrm{O}_{2}$-Verbrauch einer $E$. coli-Kultur mit AcsZusatz, so beobachtet man zunächst eine abrupte Abnahme der $\mathrm{O}_{2}$-Aufnahme, der bei 


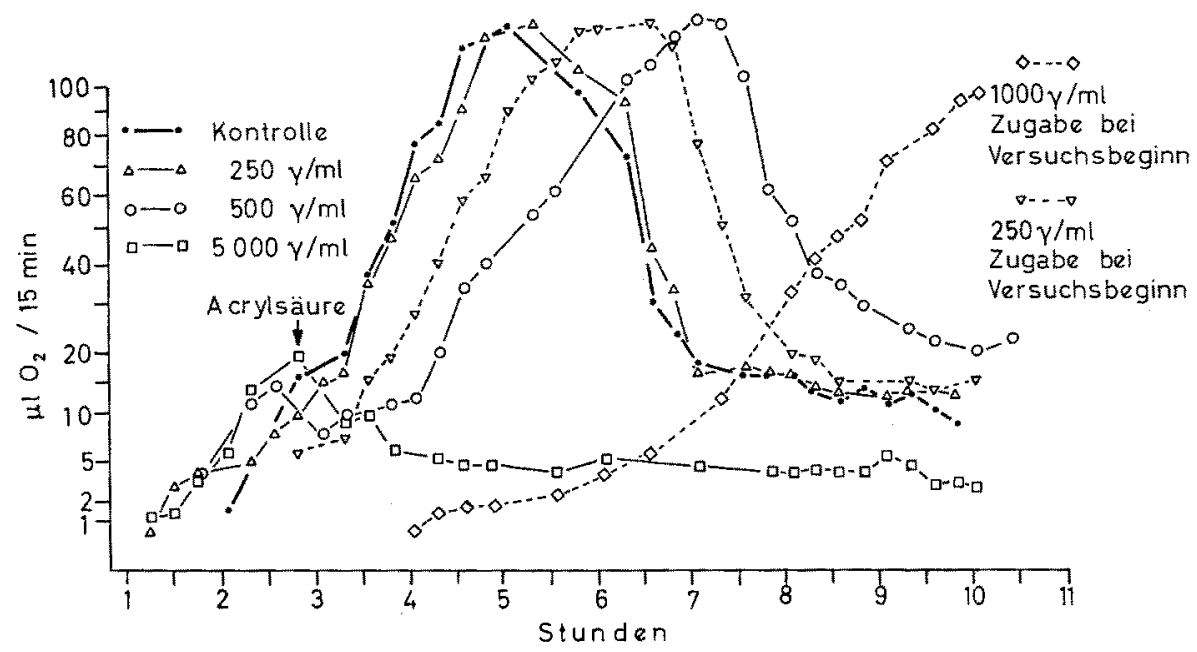

Abb. 2: $\mathrm{O}_{2}$-Verbrauch von Escherichia coli-Zellen bei Zugabe von Acrylsäure (Pfeil) zu Beginn der logarithmischen Phase oder bei Start des Versuches $(250 \mathrm{\gamma} / \mathrm{ml}$ und $500 \gamma / \mathrm{ml})$

niedrigen Acs-Konzentrationen eine teilweise oder völlige Erholung der Kultur folgt. Das Bild ähnelt somit den Beobachtungen bei der Messung der optischen Dichte der Kultur. Sehr hohe Acs-Dosierungen bringen die Atmung jedoch völlig zum Erliegen. Setzt man die Acs nicht zu Ende, sondern zu Anfang der log-Phase zu, so führen selbst sehr hohe Acs-Konzentrationen nicht zu einem völligen Abbruch des $\mathrm{O}_{2}$-Verbrauchs (Abb. 1 und 2). Impft man die Bakterien direkt in eine Acs-haltige Lösung, so beobachtet man eine Verlängerung der vorlogarithmischen Phase, und der Anstieg der Atmungskurven ist bei sehr hohen Acs-Konzentrationen etwas flacher.

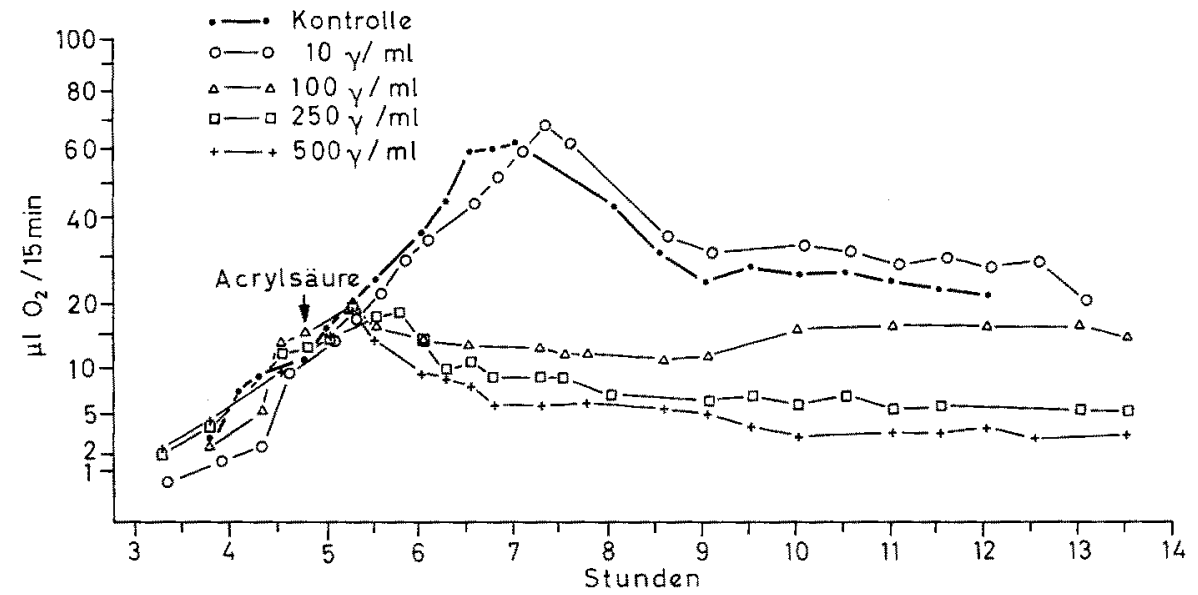

Abb. 3: $\mathrm{O}_{2}$-Verbrauch von Stapbylococcus aureus-Zellen bei Zugabe von Acrylsäure (Pfeil) zu Beginn der logarithmischen Phase 


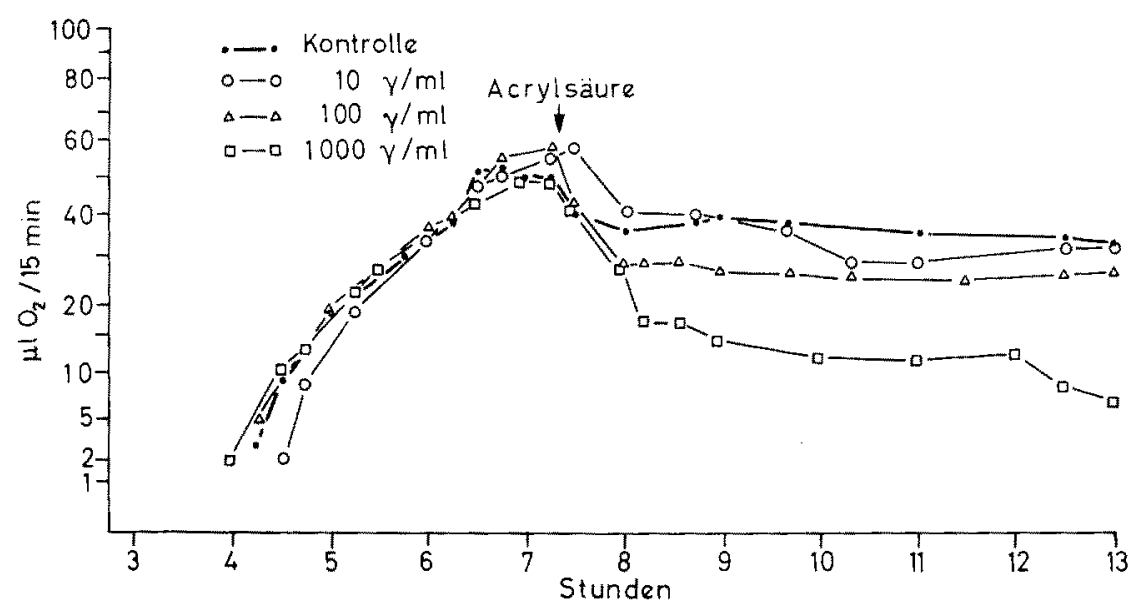

Abb. 4: $\mathrm{O}_{2}$-Verbrauch von Stapbylococcus aureus-Zellen bei Zugabe von Acrylsäure (Pfeil) bei Ende der logarithmischen Phase

Besonders bei jungen Kulturen ist die Wachstumshemmung sehr viel stärker als der Einfluß auf den $\mathrm{O}_{2}$-Verbrauch. Ab Mitte der log-Phase liegen die Werte in der gleichen Größenordnung (Heyser \& Glombrtza 1971).

Auch bei St. aureus hemmt Acs die Atmung. Im Gegensatz zu E. coli ist jedoch kein abrupter Abfall des $\mathrm{O}_{2}$-Verbrauches unmittelbar nach Acs-Zugabe zu beobachten (Abb. 3 und 4). Eine einmal wirksam gewordene Hemmung kann auch nicht wieder kompensiert werden. Der $\mathrm{O}_{2}$-Verbrauch stagniert oder sinkt sogar geringfügig ab. Der unterschiedliche Einfluß der Acs auf die Atmung der beiden Bakterienstämme spiegelt sich auch im Einfluß der Acs auf die aerobe Glykolyse wider (HEYser \& Glombitza 1971).

Der respiratorische Quotient wird durch Acs bei keinem der Versuchsorganismen. beeinflußt. Er schwankt bis zum 15 fachen der MHK innerhalb der Fehlergrenzen des Versuches von 0,98 bis 1,03 (ohne Acs 1,00).

\section{Einfluß der Acrylsäure auf die Makromolekülsynthese}

Die Synthese der Makromoleküle wurde an Hand der Einbaurate von ${ }^{14} \mathrm{C}_{(2)}$ Uracil, ${ }^{14} \mathrm{C}_{(2)}$-Thymidin und ${ }^{14} \mathrm{C}_{(\mathrm{u})}$-Leucin untersucht. Die markierten Vorstufen wurden nach unterschiedlicher Einwirkungsdauer von Acs für je 5 Minuten zugegeben, um die momentanen Syntheseraten zu erfassen. Es wurde ein E. coli-Stamm benutzt, der im Hinblick auf weitere Untersuchungen (Heyser \& Glombitza 1971) an Pyruvat adaptiert worden war (E. coli-Pyruvat-Stamm).

$75 \gamma \mathrm{Acs} / \mathrm{ml}$ entsprechen bei einer jungen Kultur vom Beginn der log-Phase $\left(\mathrm{E}_{600} 0,1\right)$ einer $\mathrm{HK}_{25}$ (Tab. 5). Der Einbau von ${ }^{14} \mathrm{C}$-Thymidin in DNS wird etwa in gleichem Maße wie das Wachstum gehemmt. Die Schwankungen erklären sich aus der niedrigen Gesamteinbaurate von nur 300-600 Imp./min. Die Proteinsynthese läuft 
während der ersten 35 min unvermindert weiter. Da die Zahl der Keime/ml in der gehemmten Probe zumindest nach $35 \mathrm{~min}$ kleiner ist als in der Kontrolle, resultiert daraus sogar eine verstärkte Proteinsynthese jeder einzelnen Zelle. Der Einbau von Uracil in RNS wird jedoch von Anfang an sehr viel stärker als alle anderen Vorgänge blockiert.

Tabelle 5

Wirkung der Acrylsäure auf die Makromolekülsynthese

\begin{tabular}{|c|c|c|c|c|c|}
\hline \multirow[b]{2}{*}{ Konzentration } & \multirow{2}{*}{$\begin{array}{c}\text { Minuten } \\
\text { nach Ver- } \\
\text { suchsbeginn }\end{array}$} & \multirow{2}{*}{$\begin{array}{l}\text { beim } \\
\text { Wachs- } \\
\text { tum }\end{array}$} & \multicolumn{3}{|c|}{$\begin{array}{r}\text { Hemmung in } \% \text { zur Kontrolle } \\
\text { beim Einbau von }\end{array}$} \\
\hline & & & $\begin{array}{l}{ }^{14} \mathrm{C}-U_{\text {racil }} \\
\text { (RNS) }\end{array}$ & $\begin{array}{c}{ }^{14} \mathrm{C} \text {-Thy- } \\
\text { midin } \\
\text { (DNS) }\end{array}$ & $\begin{array}{l}{ }^{14} \mathrm{C}-\text {-Leucin } \\
\text { (Protein) }\end{array}$ \\
\hline $\begin{array}{l}75 \text { y Acs } / \mathrm{ml} \\
\text { E. coli vom Beginn } \\
\text { der log-Phase } \\
\mathrm{E}_{600} 0,1\end{array}$ & $\begin{array}{r}0-5 \\
10-15 \\
20-25 \\
30-35\end{array}$ & $\begin{array}{l}25 \\
24 \\
27\end{array}$ & $\begin{array}{l}73 \\
68 \\
72 \\
71\end{array}$ & $\begin{array}{r}0 \\
26 \\
41 \\
32\end{array}$ & $\begin{array}{l}0 \\
0 \\
0 \\
0\end{array}$ \\
\hline 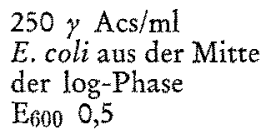 & $\begin{array}{r}0-5 \\
10-15 \\
20-25 \\
30-35\end{array}$ & $\begin{array}{l}48 \\
52 \\
50 \\
50\end{array}$ & $\begin{array}{l}90 \\
91 \\
92 \\
92\end{array}$ & $\begin{array}{r}8 \\
30 \\
39 \\
46\end{array}$ & $\begin{array}{r}5 \\
38 \\
41 \\
28\end{array}$ \\
\hline
\end{tabular}

Diese Befunde lassen sich auch bei älteren Kulturen aus der Mitte der log-Phase $\left(\mathrm{E}_{600}\right.$ ca. 0,5 ) bestätigen, die mit einer Acs-Menge versetzt wurden, die etwa einer $\mathrm{HK}_{50}$ entsprach. Hierbei zeigt sich in Ubereinstimmung mit den im Vorhergehenden geschilderten Ergebnissen, daß die Wachstumsgeschwindigkeit - gemessen an der Zunahme der optischen Dichte - ohne Latenzphase von Anfang an gehemmt wird. Das gleiche gilt auch für den Uracil-Einbau, während bei Thymidin und Leucin die Einbauraten erst nach 15 Minuten deutlich absinken.

DNP hat - ähnlich wie Acs - einen hemmenden Einfluß auf die RNS-Synthese (Simon 1966). In E. coli-Pyruvat-Zellen aus der Mitte der logarithmischen Phase wird die RNS-Synthese durch $5 \cdot 10^{-5} \mathrm{~m}$ DNP kaum gestört. $150 \gamma \mathrm{Acs} / \mathrm{ml}$ (ca. $2 \cdot 10^{-3} \mathrm{~m}$ ) stören das Wachstum kaum (Tab. 6), vermindern jedoch den Uracil-Einbau sehr stark.

Tabelle 6

Hemmung des Uracil-Einbaues bei Escherichia coli-Pyruvatstamm in Gegenwart von Acrylsäure und DNP. E. coli-Pyruvatstamm aus der Mitte der $\log -\mathrm{Ph} a \mathrm{se} \mathrm{E}_{600} 0,5$

\begin{tabular}{|cccc|}
\hline $\begin{array}{c}\text { Minuten nach } \\
\text { Versuchsbeginn }\end{array}$ & \multicolumn{3}{c|}{ Hemmung des Einbaues von ${ }^{14} \mathrm{C}$-Uracil in $\%$} \\
& $\begin{array}{c}5 \cdot 10^{-2} \mu \mathrm{Mol} \\
\mathrm{DNP} / \mathrm{ml}\end{array}$ & $\begin{array}{c}\text { zur Kontrolle bei } \\
150 \gamma \mathrm{Acs} / \mathrm{ml}\end{array}$ & $\begin{array}{r}5 \cdot 10^{-2} \mu \mathrm{Mol} \mathrm{DNP} \\
+150 \gamma \text { Acs } / \mathrm{ml}\end{array}$ \\
\hline $0-5$ & 6 & 68 & 86 \\
$10-15$ & 1 & 43 & 73 \\
$20-25$ & 5 & 46 & 68 \\
$30-35$ & 1 & 32 & 20 \\
\hline Hemmung des Wachstums & 1 & 7 & \\
nach 35 min & & & \\
\hline
\end{tabular}


Beide Substanzen zusammen wirken stark synergistisch hemmend auf das Wachstum und reduzieren den Uracil-Einbau noch einmal bis auf etwa die Hälfte.

Bei einer Fraktionierung der s-RNS und r-RNS am Saccharose-Gradienten $\mathrm{m}$-RNS wird unter den angewandten Versuchsbedingungen nicht miterfaßt - ließen sich keine signifikanten Unterschiede im Sedimentationsprofil gehemmter und ungehemmter Kulturen feststellen. Der Einbau von ${ }^{14} \mathrm{C}$-Uracil scheint in allen Fraktionen in gleicher Weise gehemmt zu sein. Eine Identifizierung der verschiedenen Peaks wurde nicht versucht. Normalerweise werden die drei ersten Peaks der r-RNS und der vierte der s-RNS zugeschrieben. Die Herkunft und Einheitlichkeit besonders der löslichen Anteile ist jedoch stark von den Versuchsbedingungen abhängig (SrMON \& PRAAG 1964, Click \& Hackett 1966).

\section{DISKUSSION}

Bereits in der Einleitung wurde gesagt, daß Acrylsäure bei unterschiedlichen Bakterienstämmen sehr verschiedenartige Stoffwechselschritte zu hemmen vermag. Diese Erfahrung konnte auch für $E$. coli und St. aureus bestätigt werden. Während der $\mathrm{O}_{2}-$ Verbrauch bei St. aureus nicht nennenswert stärker als das Wachstum gehemmt wurde, sinkt er bei $E$. coli bei höheren Acs-Konzentrationen so rapide ab, wie wir es meist nur bei bakteriziden Hemmstoffen sehen. Die Zahl der kultivierbaren Keime nimmt jedoch in der gleichen Zeitspanne von wenigen Minuten nicht ab; zudem wird der durch niedrige Acs-Gaben gesetzte Schaden bei $E$. coli rasch wieder behoben, während er bei St. aureus zeitabhängig verstärkt wird. Bei keinem Organismus wird der respiratorische Quotient im Vergleich zur Kontrolle verändert. Es ist deshalb nicht anzunehmen, daß der bei $E$. coli normalerweise vorherrschende aerobe Substratabbau auf anaerobe Energiegewinnungsprozesse umgestellt wird. Es ist wahrscheinlicher, daß der Substratabbau an einer Stelle unterbrochen wird und daß es so lange zur Anhäufung einer Zwischenstufe kommt, bis der aufgebaute Vorrat die offensichtlich reversible Hemmung überspielt. Es bleibt zu untersuchen, welche Reaktion gestört wird.

Einen ersten Hinweis gibt die Beobachtung, daß besonders SH-haltige Verbindungen Antagonisten der Acs bei $E$. coli sind, während alle anderen biologisch aktiven Substanzen keinen oder nur einen geringen Einfluß haben. Die Wirkung SH-haltiger Verbindungen kann einmal so erklärt werden, daß sie SH-Gruppen in Enzymen schützen, und zum anderen dadurch, daß die Acs durch Addition des R-SH an die Doppelbindung inaktiviert wird. Gegen den letzten Mechanismus spricht, daß alle diese Verbindungen bei $S t$. aureus gar keinen oder nur einen geringen Einfluß haben.

Es ist auffallend, daß die Atmungshemmung besonders gegen Ende des logarithmischen Wachstums sehr stark ist; im Anfang der logarithmischen Phase ist die Wachstumshemmung stärker ausgeprägt als die Verminderung der Atmung. Diese Beobachtung findet ihre Erklärung darin, daß zu Beginn der logarithmischen Phase die Syntheseleistungen die Atmungsvorgänge überwiegen. Der Einbau von Thymidin in die DNS wachsender Zellen wird insgesamt nur etwa ebenso stark gehemmt wie das Wachstum. Der Einbau von Uracil in die RNS wird jedoch sehr viel stärker unterbunden als die Zunahme der optischen Dichte. Dies könnte einmal auf Störungen der 
Uracil-Aufnahme und zum anderen auf Störungen des Uracileinbaues zurückzuführen sein. Da jedoch die RNS-Synthese auch in Zellen gehemmt ist (GLOMBITZA 1970), denen Uracil nicht über die Nährlösung zugeführt wurde, scheint Acs weder die Uracil-Synthese noch die Uracil-Aufnahme, sondern nur den Einbau in RNS zu stören. Eine Störung der m-RNS-Synthese würde sehr bald auch die Proteinsynthese mit beeinträchtigen. Der Einbau von Leucin wird jedoch gar nicht oder sehr viel weniger als das Wachstum beeinflußt.

Einen ähnlichen Einfluß auf die Synthese der Makromoleküle haben bei $E$. coli die Hemmstoffe Levorphanol (Simon \& PraAg 1964) und DNP (Simon et al. 1966). In stärkerem Maße als die Zunahme der optischen. Dichte hemmen Acs und DNP den Einbau von Uracil in $E$. coli. Es wird vermutet, daß die synergistische Hemmung der RNS-Synthese auch die Ursache der synergistischen Wachstumshemmung ist.

DNP hemmt selektiv die Synthese der r-RNS (SImon et al. 1966). Die gesamte RNS einer Zelle besteht zu einem sehr hohen Prozentsatz aus r-RNS. Acs scheint ebenfalls in die $r$-RNS-Synthese einzugreifen. Diese Vermutung wird besonders durch das Sedimentationsprofil gestützt. Die Hemmung der $r$-RNS scheint jedoch im Gegensatz zu der Wirkung des DNP nicht selektiv zu sein, da in gleichem Maße der Einbau in die lösliche Fraktion vermindert ist. Die Feststellung des genauen Angriffspunktes der Acs im RNS-Stoffwechsel muß weiteren Untersuchungen vorbehalten bleiben.

\section{ZUSAMMENFASSUNG}

1. Acrylsäure ist innerhalb der einfachen ungesättigten Carbonsäuren ein relativ spezifischer Hemmstoff für Eschericbia coli, Stapbylococcus aureus und andere Bakterien.

2. Acrylsäure hat nur in sehr hohen Konzentrationen bei sehr langen Inkubationszeiten schwach bakterizide Eigenschaften.

3. Sie hemmt besonders bei älteren $E$. coli-Zellen die Atmung sehr viel stärker als das Wachstum. Bei jungen E. coli-Kulturen und bei St. aureus sind Wachstums- und Atmungshemmung etwa gleich stark.

4. Die antibakteriallen Eigenschaften der Acrylsäure gegen $E$, coli werden durch SHhaltige Verbindungen aufgehoben. 2,4-Dinitrophenol wirkt dagegen stark synergistisch. Bei St, aureus sind diese Wirkungen nur schwach ausgeprägt.

5. Der Einbau von Leucin in Protein wird durch Acrylsäure bei $E$. coli weniger als das Wachstum, der Einbau von Thymidin in DNS fast ebenso stark wie das Wachstum und der Einbau von Uracil in RNS von Anfang an sehr viel stärker gestört. DNP steigert die Hemmung des Uracil-Einbaues durch Acrylsäure.

6. Die Synthese der r-RNS und s-RNS wird in etwa gleichem Umfang gestört; die Synthese der $m$-RNS scheint nicht beeinflußt zu werden.

Danksagungen. Herrn Prof. Dr. M. STEINER danken wir für die Unterstiitzung dieser Arbeit mit Sachmitteln des Institutes, Frl. A. WAGNER für ihre sorgfältige Mitarbeit und der DFG für eine Sachbeihilfe. Wir danken ferner Herrn Prof. Dr. W. Eschrich, Göttingen, für die Möglichkeit, seine Spinco SW $50 \mathrm{zu}$ benutzen, und Herrn Dr. Sous von der Chemie Grünenthal GmbH, Stolberg, für die Uberlassung der Bakterienstämme. 


\section{ZITIERTE LITERATUR}

Brisou, J. \& de Rautlin de la Rox, J., 1964. Action de l'acide acrylique sur St. aureus et quelques coliformes. C. r. Séanc. Soc. Biol. 158, 642-645.

Click, R. E. \& HacketT, P. D., 1966. The isolation of ribonucleic acid from plant, bacterial or animal cells. Biochim. biophys. Acta 129, 74-84.

GlombitzA, K.-W., 1968. Antimikrobielle Inhaltsstoffe in Meeresalgen. Habilitationsschrift, Bonn.

- 1969. Antibakterielle Inhaltsstoffe in Algen. 1. Mitteilung. Helgoländer wiss. Meeresunters. $19,376-384$.

HAcketT, D. P., 1960. Respiratory inhibitors. Handb. PflPhysiol. 12 (2), 21-41.

HeYser, R. \& GLOMBITZA, K.-W., 1971 (In Vorbereitung).

JANKE, A. \& DiCKSCHErT, R., 1967. Handbuch der mikrobiologischen Laboratoriumstechnik. Steinkopff, Dresden, 500 pp.

KLeIN, P., 1957. Bakteriologische Grundlagen det chemotherapeutischen Laboratoriumspraxis. Springer, Berlin, $210 \mathrm{pp}$.

LÜCK, H., 1959-1960. Einfluß von Konservierungsmitteln auf Häminenzyme. 2. Mitt. Untersuchungen über die Korrelation zwischen dem Konservierungseffekt organischer Säuren und ihrer Katalasehemmung. Z. Lebensmittelunters. u. -Forsch. 111, 190-198.

Michet, M. C., 1965. Comparison de l'action de l'acrylate de sodium et de la chlortetracycline sur la flore intestinale du porc. Annls Biol. anim. Biochim. Biophys. 5 (2), 223-236.

Rенм, H. J., 1967. Zur Kenntnis der antimikrobiellen Wirkung der Sorbinsäure. 6. Mitt. Die Wirkung der Sorbinsäure auf den Kohlehydratstoff wechsel von Escherichia coli. Zentbl. Bakt. ParasitKde. (Abt. 2) 121, 491-502.

Steburth, J. Mc. N., 1960. Acrylic acid, an "antibiotic" principle in Phaeocystis blooms in anarctic waters. Science, N.Y. 132, 676-677.

- 1961. Antibiotic properties of acrylic acid, a factor in the gastrointestinal antibiosis of polar marine animals. J. Bact. 82, 72-79.

Simon, E. J. \& PraAG, D. VAN, 1964. Inhibition of RNA synthesis in Eschericbia coli by levorphanol. Proc. natn. Acad. Sci. U.S.A. 51, 877-883.

- - \& Aronson, F. L., 1966. The selective inhibition of ribosomal RNA synthesis in Eschericbia coli by 2,4-Dinitrophenol. Molec. Pharmac. 2, 43-49.

THIJSSE, G. J. E., 1964. Fatty-acid accumulation by acrylate inhibition of $\beta$-oxidation in an alkane-oxidizing Pseudomonas. Biochim. Biophys. Acta 84, 195-197.

WhITLEY, H. R. \& ORdal, E. J., 1957. Fermentation of $\alpha$-keto-acids by Micrococcus lactilyticus. J. Bact. 74, 331-336.

Anschrift des erstgenannten Autors: Prof. Dr. K.-W. Glombrtza

Pharmakognostisches Institut

der Universität Bonn

53 Bont

Nußallee 6

Bundesrepublik Deutschland 